\title{
Inclusão do indicador "impactos ambientais" na ferramenta "Diagnóstico do Sistema Embalagem": um estudo de caso em vinícola
}

\author{
Adding the indicator "environmental impacts" in the "Diagnosis of the \\ Packaging System" tool: a case study at winery
}

\begin{abstract}
Patrícia Santos
Mestre em Processos Químicos e Bioquímicos pela Escola de Engenharia Mauá do Centro Universitário do Instituto Mauá de Tecnologia - São Caetano do Sul (SP), Brasil.
\end{abstract}

\author{
Antonio Cabral \\ Doutor em Engenharia de Produção. Professor Titular do Instituto Mauá de Tecnologia - São Caetano do Sul (SP), Brasil.
}

\begin{abstract}
Resumo
O objetivo deste estudo foi ampliar o escopo da ferramenta "Diagnóstico do Sistema Embalagem", criada para avaliar e gerenciar adequadamente o Sistema Embalagem Foram incluídos no escopo procedimentos que possibilitem avaliar impactos ambientais causados em suas operações. Aos três indicadores atuais — custo sistêmico, inovação e competências —, foi adicionado o quarto, que engloba tratamento de resíduos sólidos, emissão de gás carbônico, consumo de água e energia. Assim, o método de pesquisa empregado foi o de caráter qualitativo exploratório, por meio de um estudo de caso, a fim de verificar se a empresa participante da pesquisa tem uma visão sistêmica nas etapas do desenvolvimento do produto e, com isso, aprimorar a metodologia "Diagnóstico do Sistema Embalagem" em todo o processo que envolve a embalagem do produto escolhido. Os resultados obtidos mostram que é possível desenhar um cenário básico, a partir do qual devem ser tomadas ações estratégicas que permitirão aprimorar o desempenho do sistema em estudo. Pela sua concepção, a ferramenta pode ser adaptada a diversos produtos da empresa. A inclusão do indicador auxilia o correto posicionamento estratégico frente às políticas ambientais mundiais que estão sendo elaboradas.
\end{abstract}

Palavras-chave: gerenciamento dos impactos ambientais; visão sistêmica; embalagem.

\begin{abstract}
The objective of this study was to expand the "Diagnosis of the Packing System" tool, created to evaluate and properly manage the Packing System, adding procedures that allow the evaluation of the environmental impacts, trigged in its operations. The three current indicators are systemic cost, innovation and competences. A fourth indicator was added and comprehends the treatment of solid waste, carbon dioxide emissions, water and energy consumption. Thus, the methodology that was applied in this research was exploratory and qualitative, and it was carried out through a case study, aiming to verify if the company has a systemic perspective in the phases of the development of the product, as well as to improve the tool "Diagnosis of the Packing System" in the process that is related to the chosen product. The results of the research show that it is possible to design a basic scenario, and, through it, to plan some strategies that enable the improvement of the studied system. Through its conception, the tool can be adapted to many different products of the company. The inclusion of the indicator helps the correct strategic attitude towards the world environmental policies that have been created.
\end{abstract}

Keywords: environment impact management; systemic view; packing. 


\section{Introdução}

O mercado econômico mundial vem sofrendo intensas mudanças nas últimas décadas, que acarretam a necessidade de avaliações e reavaliações nas estratégias da gestão, para que as empresas possam se manter competitivas. Essas novas demandas abrem espaço para o uso de ferramentas que contribuem para a melhoria do desempenho organizacional. Além das questões econômicas e de qualidade em serviços, surgiu, nas últimas décadas, a preocupação com o impacto ambiental, especialmente quando se considera o uso indevido de recursos naturais, os alarmantes índices de descartes irresponsáveis e de emissões além dos níveis aceitáveis.

As embalagens têm sido consideradas grandes causadoras desse impacto. Embora estas apresentem aspectos positivos, pois reduzem as perdas de alimentos perecíveis, contribuem para alimentar pessoas nos mais variados locais no mundo e, desta forma, minimizam os impactos ambientais causados pelas perdas desses alimentos, o descarte é realizado pelo consumidor de forma irresponsável, sendo a principal causa de problemas ambientais.

Dentre as ferramentas disponibilizadas no mercado para avaliar, gerenciar e tentar minimizar os problemas na gestão de empresas, destaca-se o "Diagnóstico do Sistema Embalagem", criado por Antonio Dantas Cabral em 1994. Essa ferramenta está alicerçada em três indicadores: custo sistêmico, inovação e competências. Mesmo com os bons resultados advindos da sua utilização, observou-se a necessidade de ampliar o seu escopo, incluindo procedimentos que possibilitem avaliar os impactos ambientais - o quarto indicador —, causados por operações relacionadas ao sistema embalagem, sendo esse o objetivo deste estudo.

A fim de que o objetivo fosse alcançado, realizou-se uma pesquisa de caráter qualitativo exploratório, por meio de um estudo de caso, para verificar se a empresa participante da pesquisa tem uma visão sistêmica nas etapas do desenvolvimento do produto e, com isso, aprimorar a metodologia "Diagnóstico do Sistema Embalagem" em todo o processo que envolve a embalagem do produto escolhido.

\section{Sistema Embalagem}

Ao se pensar na evolução histórica das embalagens, constata-se que estas acompanham o homem desde a Antiguidade, quando ele utilizava elementos da natureza, como cascas e folhas, por exemplo, para condicionar, proteger e transportar os seus alimentos. Com o passar do tempo, as embalagens foram ganhando novas funções, embora mantenham, durante toda a sua história, as funções iniciais (CAVALCANTI \& CHAGAS, 2006).

A embalagem deve ser entendida como um sistema complexo, constituído de partes interdependentes, o qual pode ser definido como "o conjunto de operações, os materiais e os acessórios usados nas empresas, com o objetivo de conter, proteger, conservar e transportar seus produtos até o momento da chegada ao consumidor, considerando um custo adequado e respeitando a ética e o meio ambiente" (CABRAL \& CABRAL, 2010, p. 2).

Segundo os autores, o Sistema Embalagem (SE), representado na Figura 1, é considerado um sistema aberto, porque está sujeito a interferências do meio, como, por exemplo, inovações tecnológicas, necessidades do consumidor, variações do mercado econômico e necessidade do fabricante em atingir um determinado público. Por isso, é necessário adotar critérios de gestão que assegurem a sua sobrevivência.

O SE tem o seu início no projeto, em que devem ser verificadas as características do produto, de seu processamento, de suas necessidades de proteção, da cadeia de comercialização, bem como dos materiais e equipamentos disponíveis no mercado. A criação é simultânea ao projeto e não é considerada apenas parte dele, porque, geralmente, a responsabilidade pela sua condução é atribuída pelas empresas às agências especializadas.

Concluídos o projeto e a criação, documentam-se as características da embalagem e as variações, toleradas para ela, na etapa denominada especificação. Em seguida, há a seleção técnica e comercial de fornecedores, a compra, alicerçada em critérios técnicos e comerciais (qualidade, preço, pontualidade e assistência técnica), o recebimento e a estocagem das embalagens.

No processamento, ocorre o envase, quando "[...] a embalagem passa a se confundir com o próprio produto, assumindo a responsabilidade de contê-lo e protegê-lo até seu consumo ou utilização" (CABRAL \& CABRAL, 2010, p. 4). Por esse motivo, essa etapa deve ser monitorada por meio de indicadores estruturados e consistentes. Seguem-se, assim, a estocagem do produto acabado e a distribuição, etapa em que o produto e a embalagem passam para o ambiente externo, sendo levados até os pontos de venda. O produto e a sua embalagem estão prontos para serem adquiridos e utilizados pelo consumidor, que deve ser sempre o foco de todo o processo.

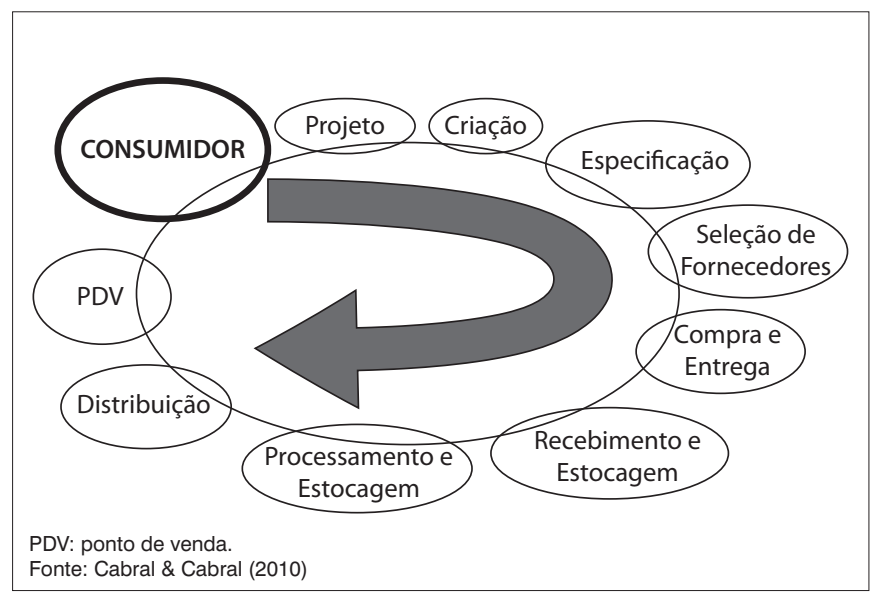

Figura 1 - Representação esquemática do Sistema Embalagem. 


\section{A Ferramenta "Diagnóstico do Sistema Embalagem"}

A ferramenta "Diagnóstico do Sistema Embalagem" foi desenvolvida por Antonio Cabral, em 1994, com o intuito de auxiliar no gerenciamento das empresas usuárias de embalagem, tendo como indicadores o custo sistêmico, a inovação e a formação de competências.

Quanto à aplicação desse método, é possível que muitas empresa afirmem que seguem o modelo, ou seja, que têm uma visão sistêmica do processo de embalagem, mas esse discurso, muitas vezes, não corresponde à realidade. No afã de parecerem preocupadas com as questões ambientais e de qualidade, maquiam o que realmente acontece no dia-a-dia da empresa. A questão de não haver correspondência entre a realidade e o que é dito se deve pela falta de alguns dados que, às vezes, são impossíveis de mensurar ou não são fidedignos.

Os dados que devem ser analisados, de acordo com os indicadores custo sistêmico, inovação e competências, propostos pela ferramenta, serão apresentados a seguir.

Deve-se considerar que custo é todo o gasto relativo aos processos industriais, como, por exemplo, matéria-prima, mão de obra e gastos gerais de fabricação, que incluem depreciação de equipamentos, energia elétrica, manutenção, materiais de escritório para fábrica, entre outros (CABRAL \& CABRAL, 2010).

Avaliar as questões relacionadas ao custo de embalagens, a partir de uma perspectiva sistêmica, significa avaliar todos os aspectos envolvidos na produção de um produto, que vai desde o seu planejamento até sua chegada ao consumidor final, considerando, já no projeto inicial, os objetivos e as estratégias para satisfazer o consumidor.

Desta forma, ao pensar no custo, é fundamental levar em consideração, dentre outros, os seguintes fatores: a escolha de materiais, a qualificação da mão de obra, a eficiência na linha de produção e a eficácia no atendimento ao cliente.

Após a coleta das informações dos custos referentes a todas as etapas do SE, esses valores são lançados na planilha da ferramenta, que consolidará automaticamente os valores finais no chamado "Quadro Consolidado" (Quadro 1).

Neste caso, conforme exemplificado, o que se observa no quadro é que o preço de compra da embalagem representa apenas 32,9\% do custo sistêmico. Ou seja, forçar o fornecedor a reduzir o seu preço pode resultar em aumento do custo sistêmico, o que prejudica o resultado operacional do usuário da embalagem.

No que se refere ao conceito de inovação, este é considerado um processo de atividades técnicas, no qual está vinculada a gestão, que resulta na comercialização de novos produtos e processos. A inovação é considerada incremental (quando o produto incorpora novos elementos em relação ao anterior, sem que sejam alteradas as suas funções básicas iniciais) ou de ruptura, caracterizando-se pela incessante busca de quebra de paradigmas, ou seja, são projetadas novas tecnologias de ruptura, como oportunidade de aprendizagem e melhora na competitividade.
Quanto à inovação, a ferramenta "Diagnóstico do Sistema Embalagem" propõe que a coleta das informações, para avaliar o gerenciamento dos investimentos nesta área, compreende os dois anos anteriores ao período da pesquisa e os dois posteriores a ela. Esse panorama permite uma significativa avaliação do que foi realizado até o momento e das expectativas futuras, possibilitando a reavaliação das estratégias de investimento e possíveis melhorias na produção. No Quadro 2, tem-se um exemplo de avaliação do investimento em inovações e tecnologia no SE.

No que tange ao indicador "avaliação das competências", de acordo com a ferramenta "Diagnóstico de Sistema Embalagem", a qualificação técnica da equipe, envolvida com o sistema, é imprescindível, pois somente um grupo qualificado é capaz de diagnosticar as incertezas do mercado, bem como saber detectar e aproveitar as oportunidades que surgem, colaborando, assim, com o objetivo de levar a empresa a ocupar um lugar privilegiado junto aos seus concorrentes.

O Quadro 3 ilustra a proposta de consolidação dos dados de atividades de formação de competências dos funcionários que trabalham no SE. Esse indicador mede o número de horas efetivamente utilizadas pelos profissionais envolvidos no SE em treinamento, cursos, feiras e exposições, visitas técnicas a fornecedores, entre outros. O valor referência adotado é 8 h/mês (4\% das horas trabalhadas) de treinamento, com o mínimo necessário para consolidar e equalizar o conhecimento adquirido pelos funcionários e transformá-lo em competências.

\section{Ponto crítico da ferramenta "Diagnóstico do Sistema Embalagem"}

Mesmo que a ferramenta "Diagnóstico do Sistema Embalagem" tenha sido elaborada com o objetivo de possibilitar um gerenciamento eficiente e eficaz do sistema, não contemplou o indicador "impacto ambiental". Pode-se dizer que essa necessidade é determinante,

Quadro 1 - Exemplo de dados consolidados de custo sistêmico.

\begin{tabular}{|c|c|c|}
\hline & Valor & Unidade \\
\hline Quantidade de produto & 1.650 .000 & Unidades \\
\hline Período considerado & 1 & Mês \\
\hline \multirow{2}{*}{ ETAPA } & \multicolumn{2}{|c|}{ CUSTO R\$ } \\
\hline & TOTAL & UNIDADE \\
\hline Compras & $559.625,00$ & 0,3392 \\
\hline Estocagem de materiais & $5.120,16$ & 0,0031 \\
\hline $\begin{array}{l}\text { Envase/encaixotamento/paletiza- } \\
\text { ção }\end{array}$ & $359.790,63$ & 0,2181 \\
\hline Estocagem de produto acabado & $342.225,00$ & 0,2074 \\
\hline Distribuição & $229.350,00$ & 0,1390 \\
\hline Pontos de venda & $203.362,50$ & 0,1223 \\
\hline Total & $1.699 .473,28$ & 1,0300 \\
\hline Custo sistêmico, $\mathrm{R} \$ / \mathrm{um}$ & 1,030 & \\
\hline Preço de compra & 0,339 & $32,90 \%$ \\
\hline
\end{tabular}

Fonte: Ferramenta Diagnóstico Embalagem (CABRAL, 1994) 
visto que, na história mundial, por meio de estudos e pesquisas, observa-se que qualquer ação de gestão proposta em qualquer segmento da sociedade tem de considerar a produção sustentável.

\section{Modelo}

A questão que norteou a construção deste estudo é a seguinte: Como ampliar o escopo da ferramenta "Diagnóstico do Sistema Embalagem", para incluir indicadores de impacto ambiental, causados por materiais, acessórios e operações relacionadas à embalagem? Para atingir o objetivo desta pesquisa, foi construído o roteiro para o quarto indicador da ferramenta "Diagnóstico do Sistema Embalagem", para uso em empresas de pequeno e médio porte, o qual avalia o funcionamento da gestão do SE, a partir de uma perspectiva sistêmica. Para isso, o roteiro foi elaborado mediante as premissas defendidas pela Produção Mais Limpa ${ }^{1}$. Cabe salientar que também foi considerado o contexto da empresa na qual se realizou o estudo.

O roteiro para a entrevista e posterior organização dos dados foi dividido em quatro categorias: Resíduos Sólidos, Emissões, Energia e Água. Na categoria Resíduos Sólidos, o roteiro está alicerçado no Programa de Política Nacional de Resíduos Sólidos (PNRS) - 2010, especificamente no artigo 14, para a avaliação do gerenciamento dos resíduos sólidos. Esse artigo é composto por planos de atuação e requisitos mínimos para o gerenciamento adequado de resíduos em indústrias, em serviços de saúde, rurais, especiais ou diferenciados. No que se refere à gestão de emissões de gases e efeito estufa (GEE),

Quadro 2 - Exemplo de avaliação do investimento em inovações e tecnologia no Sistema Embalagem.

\begin{tabular}{|c|c|c|c|c|c|c|}
\hline \multirow{2}{*}{ Etapa } & \multirow{2}{*}{ Inovação } & \multicolumn{2}{|c|}{ Investimento } & \multicolumn{3}{|c|}{ Benefícios } \\
\hline & & $\mathrm{R} \$$ & $\%$ s/fat. & $\mathrm{R} \$$ & $\%$ s/fat. & Intangíveis \\
\hline \multicolumn{7}{|l|}{ Consumidor } \\
\hline \multicolumn{7}{|l|}{ Projeto } \\
\hline \multicolumn{7}{|l|}{ Especificação } \\
\hline \multicolumn{7}{|l|}{$\begin{array}{l}\text { Avaliação de } \\
\text { fornecedores }\end{array}$} \\
\hline \multicolumn{7}{|l|}{ Recebimento } \\
\hline \multicolumn{7}{|c|}{ Estocagem embalagem } \\
\hline \multicolumn{7}{|l|}{ Linha de envase } \\
\hline \multicolumn{7}{|c|}{ Estocagem produto } \\
\hline \multicolumn{7}{|l|}{ Distribuição } \\
\hline Pontos de venda & & & & & & \\
\hline
\end{tabular}

$\%$ s/fat:: informar o valor de benefício sobre a porcentagem do faturamento da empresa.

Fonte: Cabral \& Cabral (2010).

Quadro 3 - Consolidação dos dados relativos à competência.

\begin{tabular}{|c|c|c|c|c|c|c|c|c|c|}
\hline \multirow{3}{*}{ ETAPA DO SE } & \multicolumn{9}{|c|}{ GRAU DE ATUALIZAÇÃO (horas, mês/pessoa) } \\
\hline & \multirow[t]{2}{*}{ Profissional } & \multicolumn{2}{|c|}{ Cursos } & \multicolumn{2}{|c|}{ Visitas técnicas } & \multicolumn{2}{|c|}{ Livros e revistas } & \multicolumn{2}{|c|}{ Feiras } \\
\hline & & Descrição & $\mathrm{h}$ & Descrição & $\mathrm{h}$ & Descrição & $\mathrm{h}$ & Descrição & $\mathrm{h}$ \\
\hline \multicolumn{10}{|l|}{ Consumidor } \\
\hline \multicolumn{10}{|l|}{ Projeto } \\
\hline \multicolumn{10}{|l|}{ Criação } \\
\hline \multicolumn{10}{|l|}{ Especificação } \\
\hline \multicolumn{10}{|c|}{ Avaliação de fornecedores } \\
\hline \multicolumn{10}{|l|}{ Compras } \\
\hline \multicolumn{10}{|l|}{ Recebimento } \\
\hline \multicolumn{10}{|c|}{ Estocagem embalagem } \\
\hline \multicolumn{10}{|l|}{ Linha de envase } \\
\hline \multicolumn{10}{|c|}{ Estocagem produto } \\
\hline \multicolumn{10}{|l|}{ Distribuição } \\
\hline Pontos de venda & & & & & & & & & \\
\hline
\end{tabular}

SE: Sistema Embalagem.

Fonte: Cabral \& Cabral (2010)

${ }^{1}$ Produção Mais Limpa é "a aplicação contínua de uma estratégia econômica, ambiental e tecnológica integradas aos processos e produtos, a fim de aumentar a eficiência no uso de matérias primas, água e energia, através da não-geração, minimização ou reciclagem de resíduos gerados em um processo produtivo. Produção Mais Limpa também pode ser chamada de 'Prevenção da Poluição"' (FERNANDES et al., 2001, p. 5). 
o instrumento tem como fundamentação as metodologias sugeridas pelo Programa Brasileiro Greenhouse Gas (GHG) Protocol. Quanto à questão do uso da energia, o roteiro está alicerçado no Programa SEBRAE de Eficiência Energética (SEBRAE, 2003), organizado em cinco cartilhas, as quais oferecem conceitos básicos de energia, o uso adequado da energia elétrica e as estratégias que podem proporcionar a redução do consumo, diminuindo, por conseguinte, os impactos gerados pelo consumo inadequado da mesma.

Em relação à água, o roteiro segue as diretrizes sugeridas pela Produção Mais Limpa.

O levantamento das informações seguiu os passos apresentados a seguir.

Levantar as informações seguindo o roteiro de entrevista, com questões semiestruturadas, elaboradas com o objetivo de verificar o funcionamento do SE vigente na empresa, atribuindo os conceitos apresentados nas Tabelas 1 a 4. Para cada resposta obtida na entrevista, deve-se atribuir um valor de 1 a 3 , sendo que será atribuído o valor 3 às empresas que seguirem as orientações para Produção Mais Limpa e as diretrizes e/ou as recomendações de programas de gestão e/ou legislações nacionais ou municipais; valor 2, para as empresas que atenderem parcialmente às recomendações para Produção Mais Limpa e as diretrizes das legislações nacionais e/ou municipais ou algum programa de gestão; finalmente, será atribuído valor 1 para as empresas que não seguem as orientações para Produção Mais Limpa e as diretrizes ou as orientações das legislações nacionais e/ou municipais vigentes para cada categoria, bem como programas de gestão. As Tabelas 1 a 4 referem-se aos conceitos para a avaliação dos parâmetros relacionados aos resíduos sólidos, às emissões de gás carbônico, ao consumo de energia e ao consumo de água.

\section{Definição dos pesos}

Os pesos devem estar de acordo com a área de atuação da empresa, como se segue:

- àqueles indicadores que não possuem relevância dentro da área de atuação da empresa, é atribuído peso 1;

- para indicadores com grau de importância intermediário, é atribuído peso 2;

- indicadores que possuem importância determinante em relação aos demais recebem peso 3 .

As verificações e os atributos devem ser organizados, conforme o Quadro 4.

Consolidadas as médias na tabela, constrói-se o gráfico do indicador ambiental, conforme ilustrado na Figura 2.

\section{Metodologia}

O método de pesquisa empregado foi o de caráter qualitativo exploratório, por meio de um estudo de caso, a fim de verificar se a empresa escolhida para participar do estudo tem uma visão sistêmica nas etapas do desenvolvimento do produto escolhido e, com isso, aprimorar a metodologia "Diagnóstico do Sistema Embalagem" e, em um todo, o processo que envolve a embalagem.

\section{Variáveis da questão da pesquisa}

As variáveis levadas em consideração neste estudo foram as relacionadas ao "Diagnóstico do Sistema Embalagem" (custo sistêmico, inovação e competências), bem como às variáveis incluídas

Tabela 1 - Conceitos para avaliação dos parâmetros relacionados aos Resíduos Sólidos.

$\begin{array}{ll}\text { Conceito } & \text { Definição } \\ 1 & \begin{array}{l}\text { Para empresas que não possuem projetos e/ou planos } \\ \text { de ação que atendam às orientações para Produção Mais } \\ \text { Limpa e às diretrizes do PNRS e/ou legislação municipal }\end{array} \\ 2 & \begin{array}{l}\text { Para empresas que atendem parcialmente às orientações } \\ \text { para Produção Mais Limpa e às diretrizes do PNRS }\end{array} \\ 3 & \begin{array}{l}\text { Para empresas que possuem projetos e planos de ação que } \\ \text { seguem as orientações para Produção Mais Limpa e PNRS } \\ \text { e/ou legislação municipal }\end{array}\end{array}$

PNRS: Política Nacional de Resíduos Sólidos.

Tabela 2 - Conceitos para avaliação dos parâmetros relacionados a emissões de gás carbônico.

$\begin{array}{ll}\text { Nota } & \text { Definição } \\ 1 & \begin{array}{l}\text { Para empresas que não possuem projetos ou planos de ação } \\ \text { para as orientações da Produção Mais Limpa e do GHG Protocol }\end{array} \\ 2 & \begin{array}{l}\text { Para as empresas que atendem, parcialmente, às recomenda- } \\ \text { ções da Produção Mais Limpa e do GHG Protocol }\end{array} \\ & \begin{array}{l}\text { Para as empresas que possuem projetos que aplicam as orien- } \\ \text { tações para Produção Mais Limpa e as recomendações do GHG } \\ \text { Protocol }\end{array}\end{array}$

GHG: Greenhouse Gas.

Tabela 3 - Conceitos para avaliação dos parâmetros relacionados ao consumo de energia.

\begin{tabular}{ll} 
Nota & Definição \\
1 & $\begin{array}{l}\text { Para as empresas que não possuem projetos e planos de ação } \\
\text { que atendam às recomendações para Produção Mais Limpa e } \\
\text { consumo inteligente de energia }\end{array}$ \\
$2 \quad \begin{array}{l}\text { Para as empresas que atendem parcialmente às recomendações } \\
\text { para Produção Mais Limpa e consumo inteligente de energia }\end{array}$ \\
$\begin{array}{l}\text { Para as empresas que possuem projetos e planos de ação que } \\
\text { atendam às recomendações para a Produção Mais Limpa e } \\
\text { consumo inteligente de energia }\end{array}$ \\
\hline
\end{tabular}

Tabela 4 - Conceitos para avaliação dos parâmetros relacionados ao consumo de água.

Nota $\begin{aligned} & \text { Definição } \\ & \text { Para as empresas que não possuem projetos e planos de ação } \\ & \text { que atendam às recomendações para Produção Mais Limpa e às } \\ & \text { orientações para consumo de água }\end{aligned}$
$\begin{aligned} & \text { Para as empresas que atendem parcialmente às recomendações } \\ & \text { para Produção Mais Limpa e às orientações para consumo de água }\end{aligned}$
$\begin{aligned} & \text { Para as empresas que possuem projetos e planos de ação que } \\ & \text { atendam às recomendações para Produção Mais Limpa e às } \\ & \text { orientações para consumo de água }\end{aligned}$


pelo indicador ambiental, além da aplicação contínua de uma estratégia econômica, ambiental e tecnológica integradas aos processos e produtos. Foram levados também em consideração os indicadores propostos nos Manuais de Produção Mais Limpa².

\section{Procedimentos de coleta de dados}

Os dados foram coletados mediante entrevistas — baseadas em questionário semiestruturado, que indaga sobre o funcionamento do SE vigente na empresa - e durante diversas visitas à empresa.

O roteiro para coleta de dados foi o seguinte:

1) levantamento de informações sobre o funcionamento do SE na empresa, referentes aos indicadores que constituem atualmente a ferramenta utilizada neste estudo: custo sistêmico, inovação e competência;

Quadro 4 - Registro das avaliações dos indicadores de impacto ambiental.

\begin{tabular}{|c|c|c|c|}
\hline Categorias & Indicador & Nota & Média \\
\hline \multirow{12}{*}{ Resíduos Sólidos } & RS01 & 6 & \multirow{12}{*}{6,9} \\
\hline & RS02 & 9 & \\
\hline & RS03 & 9 & \\
\hline & RSO4 & 3 & \\
\hline & RS05 & 3 & \\
\hline & RS06 & 9 & \\
\hline & $\mathrm{RS} 07$ & 6 & \\
\hline & RS08 & 9 & \\
\hline & RS09 & 9 & \\
\hline & RS10 & 9 & \\
\hline & RS11 & 9 & \\
\hline & RS12 & 2 & \\
\hline \multirow{7}{*}{ Emissões } & EM01 & 6 & \multirow{7}{*}{3,4} \\
\hline & EM02 & 3 & \\
\hline & EM03 & 3 & \\
\hline & EM04 & 3 & \\
\hline & EM05 & 3 & \\
\hline & EM06 & 3 & \\
\hline & EM07 & 3 & \\
\hline \multirow{4}{*}{ Energia } & E01 & 3 & \multirow{4}{*}{3,0} \\
\hline & E02 & 3 & \\
\hline & E03 & 3 & \\
\hline & E04 & 3 & \\
\hline \multirow{4}{*}{ Água } & A01 & 3 & \multirow{4}{*}{5,3} \\
\hline & A02 & 3 & \\
\hline & A03 & 6 & \\
\hline & A04 & 9 & \\
\hline \multicolumn{4}{|l|}{ Pesos atribuídos } \\
\hline RS01 a RS11 & 3 & & \\
\hline RS12 & 2 & & \\
\hline EM01 a EM07 & 3 & & \\
\hline E01 a E04 & 3 & & \\
\hline $\mathrm{A} 01 \mathrm{a} \mathrm{A} 04$ & 3 & & \\
\hline
\end{tabular}

2) levantamento de informações referentes ao impacto ambiental gerado pela embalagem do produto escolhido, para identificar indicadores que possam ser utilizados no modelo;

3) validação do modelo na empresa;

4) análise dos dados e possível sugestão de uso do modelo para outras cadeias produtivas.

Os dados coletados referentes ao quarto indicador (impacto ambiental), objetivo principal deste trabalho, buscam a expectativa de que o peso varie entre 2,0 e 3,0 para todos os itens e de que a nenhum deles seja atribuído o peso 1 .

\section{Procedimentos para análise dos dados coletados}

Os dados obtidos, referentes ao custo sistêmico, inovação e formação de competências, foram analisados e classificados segundo os procedimentos recomendados pela ferramenta "Diagnóstico do Siste ma Embalagem".

As informações referentes ao impacto ambiental foram analisadas segundo a proposta detalhada na seção O Modelo.

\section{Considerações finais}

O modelo proposto foi aplicado experimentalmente em uma empresa produtora de vinhos e espumantes, onde foram coletados e analisados dados, permitindo a adequada compreensão da situação de empresa sob a ótica dos quatro indicadores utilizados - custo sistêmico, inovação, competências e impacto ambiental.

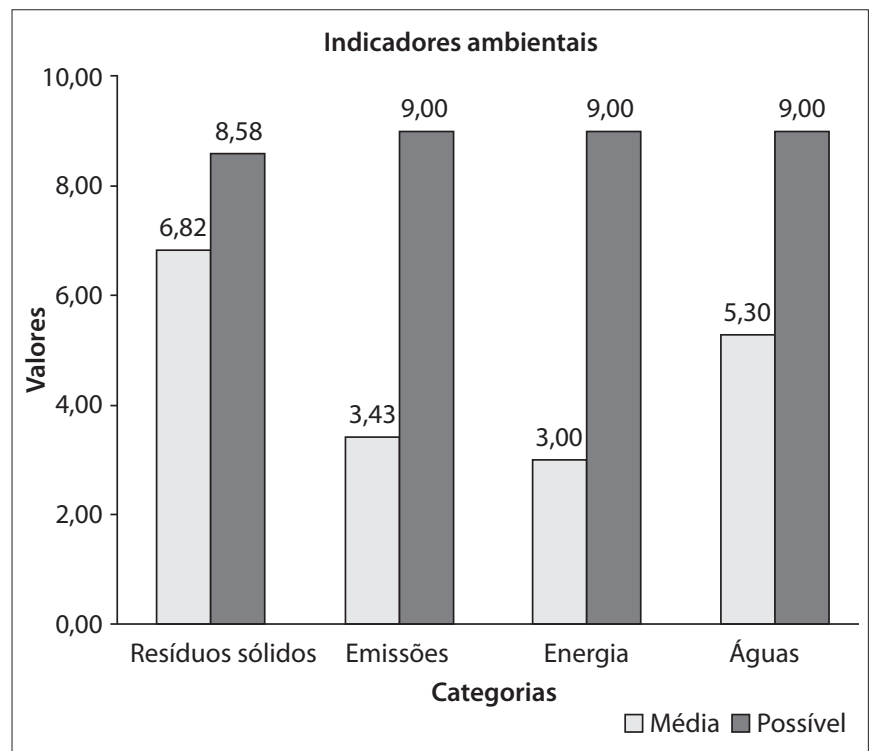

Figura 2 - Indicadores ambientais.

\footnotetext{
${ }^{2}$ Quantidade de resíduos sólidos gerados por unidade de produto acabado; de resíduos reciclados por quantidade de substância equivalente considerada; número de reclamações da comunidade; tempo; consumo de água por número de empregados; insumos utilizados (quantidade e custo de aquisição); equipamentos utilizados no(s) processo(s) produtivo(s); fontes de abastecimento e finalidades do uso da água e tipo de tratamento utilizado; locais de armazenamento e formas de acondicionamento; existência ou não de emissões atmosféricas e sistemas de controle utilizados; custos relativos ao controle dos resíduos gerados (armazenamento, tratamento e transporte); volume de produtos produzidos.
} 
Em relação ao primeiro indicador (custo sistêmico), foi identificado um equilíbrio entre o valor do custo sistêmico e material de embalagem, embora tenham sido sugeridas medidas para redução das perdas, identificadas, principalmente, na falta de controle sobre alguns itens envolvidos no processo.

O principal resultado observado no que tange ao segundo indicador (inovação) demonstra um investimento mais significativo no produto do que na embalagem. $\mathrm{O}$ investimento foi direcionado às campanhas e aos rótulos. Acredita-se, portanto, que a empresa poderia investir em inovações no SE, especialmente por serem, também, uma ferramenta de marketing.

Assim como no indicador inovação, observa-se, em relação ao terceiro indicador (competências), o foco de investimento mais uma vez no produto e não na embalagem. As participações em cursos, feiras e eventos em geral buscam o aperfeiçoamento do produto, mas não das embalagens. As informações acerca de embalagens são obtidas por meio de revistas, disponibilizadas no setor de qualidade da empresa. Com o objetivo de aumentar a rentabilidade da empresa, o presente estudo tem demonstrado estratégias a serem adotadas para esse aumento. Entende-se, portanto, que seria importante o investimento em pessoal com formação técnica para o segmento de embalagens. Os diversos profissionais ligados, de alguma forma, ao sistema de embalagens da empresa não possuem formação específica para o setor, o que indica, ao menos, a necessidade de cursos de capacitação para esses funcionários.

O foco deste trabalho, a categoria impacto ambiental, demonstrou, em termos gerais, que a empresa tem conhecimento acerca das leis sobre Resíduos Sólidos. O estudo evidenciou que as práticas da empresa estão em consonância com as diretrizes do Programa Nacional de Resíduos Sólidos, como, por exemplo, destinar de forma ambientalmente adequada os rejeitos, mapear e monitor o destino dado aos resíduos e promover a educação ambiental. Além de seguir as diretrizes do Programa de Política Nacional de Resíduos Sólidos, a empresa segue as normas da Associação Brasileira de Normas Técnicas (ABNT) para segregação, identificando e classificando os resíduos.

Por outro lado, ainda em relação aos Resíduos Sólidos, sugeriu-se à empresa que busque orientar os seus consumidores sobre a responsabilidade dos mesmos, no que se refere ao descarte das embalagens, estabelecendo, assim, a responsabilidade compartilhada. Adotar medidas de política reversa e monitorar a atuação de seus fornecedores em relação à destinação dos resíduos sólidos gerados por eles também ajudaria a empresa a se enquadrar ao previsto no PNRS - 2010.

Em relação às emissões, foi possível constatar que a empresa não possui nenhum sistema de controle. Em função disso, sugeriu-se que sejam adotadas as diretrizes do GHG Protocol, para mapeamento, monitoramento e controle dos dados. Outro item para o qual a empresa não possui sistema de controle é o de energia. Sem monitoramento do consumo de energia, não há como estabelecer metas para redução. Paralelamente à não existência de metas de redução de energia, a empresa também não possui fontes de energia alternativa, nem dados sobre a eficiência energética de seus processos. É importante, portanto, que a empresa busque informações e estratégias para o uso inteligente da energia.

O consumo de água da empresa estudada também não possui controle. Embora adote medidas de reutilização da água, a falta de controle sobre o consumo acarreta impossibilidade de metas de redução no consumo. A empresa trata toda a água utilizada por meio da sua própria Estação de Tratamento, construída de forma ecologicamente correta, de acordo com as normas da Fundação Estadual de Proteção Ambiental (FEPAM). Nesse quesito, foi sugerida a implantação do controle do consumo de água, a fim de atender às orientações da Produção Mais Limpa e, assim criar metas de redução do consumo.

A partir do levantamento realizado neste estudo, foram identificados pontos positivos e outros que deverão ser incluídos em uma extensa pauta de otimizações de gerenciamento do SE. Esse fato, por si só, valida a aplicabilidade do "Diagnóstico do Sistema Embalagem" na sua forma estendida, com a inclusão do indicador impacto ambiental.

O trabalho atendeu ao objetivo inicialmente proposto, porque ampliou o escopo da metodologia "Diagnóstico do Sistema Embalagem", incluindo procedimentos que possibilitem avaliar os impactos ambientais, causados pelas operações relacionadas às embalagens, e aprimorando seu uso no gerenciamento de sistemas de embalagem.

A partir da consolidação dos dados, foi possível constatar — no que se refere ao custo sistêmico, inovações e competências - que a empresa estudada não adota a gestão sistêmica das questões relacionadas à embalagem. Os métodos de controle, utilizados pela empresa no período analisado, não oferecem as informações necessárias para que possam ser verificadas possíveis falhas no sistema. Isso denota que o ambiente analisado ainda requer trabalho adicional, a fim de dar-lhe a consistência necessária para a adequada gestão do seu SE.

Os resultados permitem inferir que o indicador criado para a ferramenta é consistente e poderá ser aplicado em empresas de outros setores. Além disso, a pesquisa mostrou que o uso da ferramenta força os gestores a avaliar claramente a situação atual do gerenciamento do SE das empresas; orienta o entendimento do SE em empresas nas quais os indicadores podem não estar disponíveis ou não serem confiáveis, porque incentiva a intensa verbalização; facilita o entendimento da estrutura interna da empresa mediante a ótica do SE e permite planejar o seu futuro modus operandi; cria uma linguagem comum a todas as empresas, facilitando, assim, a tomada de decisões orientadas para os seus objetivos. 


\section{Referências}

BRASIL. Ministério do Meio Ambiente. (2010) Política Nacional de Resíduos Sólidos. Lei 12.305/2010. Relatório Preliminar: Minuta final. Brasília, 2002, versão II. Disponível em: <http://www.planalto. gov.br/ccivil_03/_ato2007-2010/2010/lei/l12305.htm>. Acesso em: 5 nov. 2010.

CABRAL, A.C.D. (1994) Qualidade total em sistemas de embalagem para alimentos. In: CONGRESSO BRASILEIRO DE CIÊNCIA E TECNOLOGIA DE ALIMENTOS - SBCTA, 14., 1994. São Paulo.

CABRAL, A.C.D. \& CABRAL, C.B. (2010) Qualidade total no sistema embalagem. São Paulo: Centro Universitário do Instituto Mauá de Tecnologia
CAVALCANTI, P. \& CHAGAS, C. (2006) História da embalagem no Brasil. São Paulo: Grifo Projetos Históricos e Editoriais.

FERNANDES, J.V.G. GONÇALVES, E.; ANDRADE, J.C.S.; KIPERSTOK, A. (2001) Introduzindo práticas de produção mais limpa em sistemas de gestão ambiental certificáveis: uma proposta prática. Engenharia Sanitária e Ambiental, v. 6, n. 3, p. 157-164.

Programa Brasileiro GHG Protocol. Disponível em: <http://www. ghgprotocolbrasil.com.br/index.php? $r=$ site/CapaSecao\&id $=1>$. Acesso em: 23 ago. 2010.

Serviço Brasileiro de Apoio às Micro e Pequenas Empresas (SEBRAE). (2003) Uso Inteligente da Energia. Cartilhas 1, 2, 3, 4 e 5. Brasília: SEBRAE. 